\title{
Distribution of glutathione S-transferase isoenzymes in human kidney: basis for possible markers of renal injury
}

\author{
D J HARRISON，R KHARBANDA，D SCOTT CUNNINGHAM，L I MCLELLAN,* \\ J D HAYES*
}

Department of Pathology, University of Edinburgh Medical School, Edinburgh, and the *University Department of Clinical Chemistry, Royal Infirmary, Edinburgh

SUMMARY To determine whether the tissue distribution of glutathione S-transferase (GST) isoenzymes could define the precise nature of renal injury, 13 adult kidneys were studied, using specific antibodies raised against purified isoenzymes. Basic GST stained strongly proximal convoluted tubules and some medullary tubules; acidic GST stained strongly distal convoluted tubules and medullary tubules; neutral GST stained similarly to acidic GST, but weaker, and microsomal GST stained glomerular and interstitial endothelium and collecting ducts deep in the medulla, although there was considerable variation in staining intensity among cases.

It is suggested that the measurement of these isoenzymes in serum and urine may help to eiucidate the localisation of tissue damage, which may be particularly valuable in patients with cyclosporine toxicity following renal transplantation.

The release of cytoplasmic enzymes during all injury, and their detection in serum, although not specific, is of established value in the investigation of liver and cardiac diseases. Clearly, a similar battery of enzymes whose release could be related to different forms of renal injury would be of great clinical importance.

The glutathione S-transferases (GST) comprise a multigene family of detoxification enzymes which are present in many organs and are expressed in specific tissue. ${ }^{12}$ These enzymes are important in the conjugation of many electrophilic substances, including cytotoxic drugs, herbicides, and carcinogens, with reduced glutathione. ${ }^{1-3}$ In man there are three classes of cytosolic isoenzyme: the acidic, basic, and neutral GSTs; these classes of GST are structurally and functionally distinct. ${ }^{4}$ In addition to the cytosolic forms, a distinct microsomal GST has been described. ${ }^{5}$

Basic GST, formerly known as ligandin, is present in the proximal convoluted tubules in rabbit and man. ${ }^{67}$ This class of GST is not normally present in urine ${ }^{8}$ but appears in various forms of tubular injury including ischaemia, ${ }^{9}$ Cis-platinum toxicity, ${ }^{8}$ gentamicin toxicity $^{10}$ and after exposure to heavy metal."

Both acidic and neutral GSTs have been detected in

Accepted for publication 19 January 1989 the human kidney using column chromatography to analyse renal extracts, ${ }^{1213}$ but the precise localisation of these enzymes in kidney tissue is unknown. If the tissue distribution of these isoenzymes in kidney differs then the ratio of one isoenzyme to another may help to further clarify the precise nature of the renal injury. This study examines the intrarenal distribution of cytosolic and microsomal GST by immunohistochemical analysis using specific polyclonal antisera raised against purified human GST.

\section{Material and methods}

Thirteen formalin fixed adult kidneys were studied. Eleven of these were obtained from tumour nephrectomy specimens where the tumour was small and caused no clinically important compression of renal parenchyma. Two kidneys were obtained at necropsy and these were normal histologically.

The cytosolic enzymes, acidic, basic, and neutral, and the microsomal enzyme were isolated and purified as previously described. ${ }^{41415}$ Rabbits were injected subcutaneously with $200 \mu \mathrm{g}$ of purified GST in complete Freund's adjuvant. After six weeks a repeat injection in incomplete Freund's adjuvant was given. The antibody specificity and titre were determined by Western blot analysis and radioimmunoassay. None 
Table Summary of staining with antibodies to isoenzymes of GST

\begin{tabular}{lllllll}
\hline Isoenzyme & Glomeruli & PCT* & DC $\dagger$ & Medulla & Vessels & Calyx \\
\hline Basic & - & & ++++ & + & - & ++ \\
Acidic & ++ & + & ++++++ & + muscle & ++ \\
$\begin{array}{l}\text { Podocyte } \\
\begin{array}{l}\text { Meutral } \\
\text { Micro- } \\
\text { somal }\end{array}\end{array}$ & \pm & ++ & + & - & ++ \\
\hline
\end{tabular}

*PCT, proximal convoluted tubule.

†DCT distal convoluted tubule.

of the antibodies to GST isoenzymes cross reacted with any other isoenzymes.

The rabbit polyclonal antisera to GST were incubated with paraffin wax sections for one hour, diluted 1/200 in phosphate buffered saline. After washing, antibody binding was detected using biotinylated antirabbit IgG (Dako) and a streptavidin

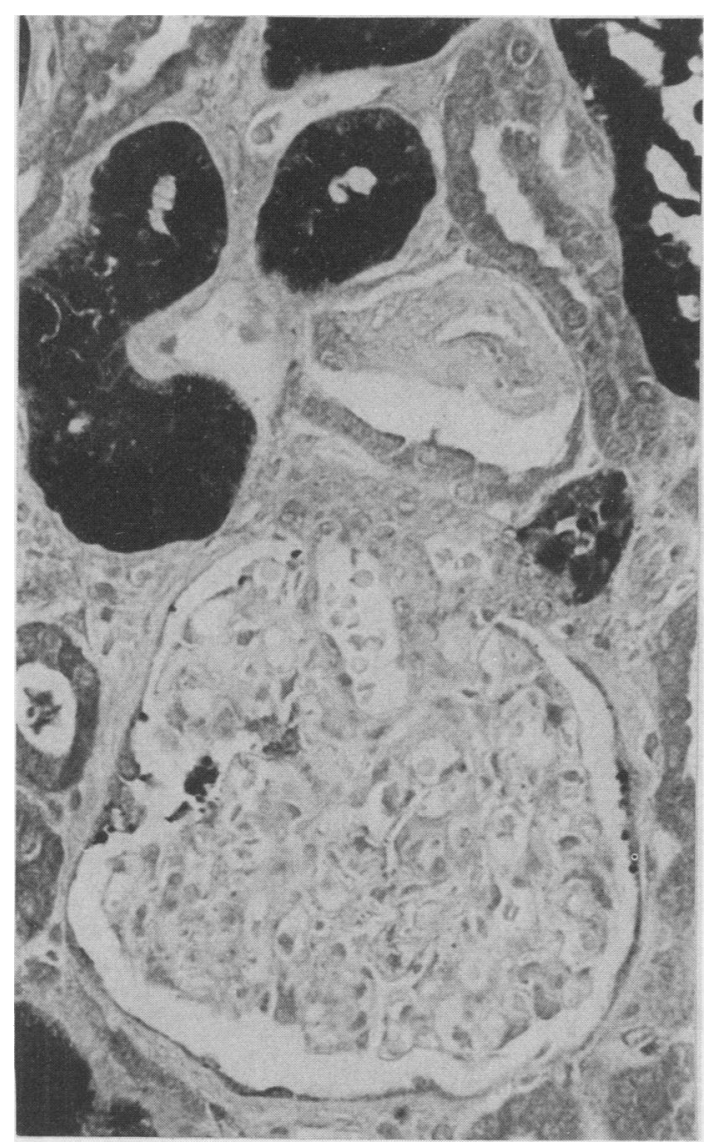

Fig 1 Photomicrograph showing intense staining in proximal tubules for basic GST.

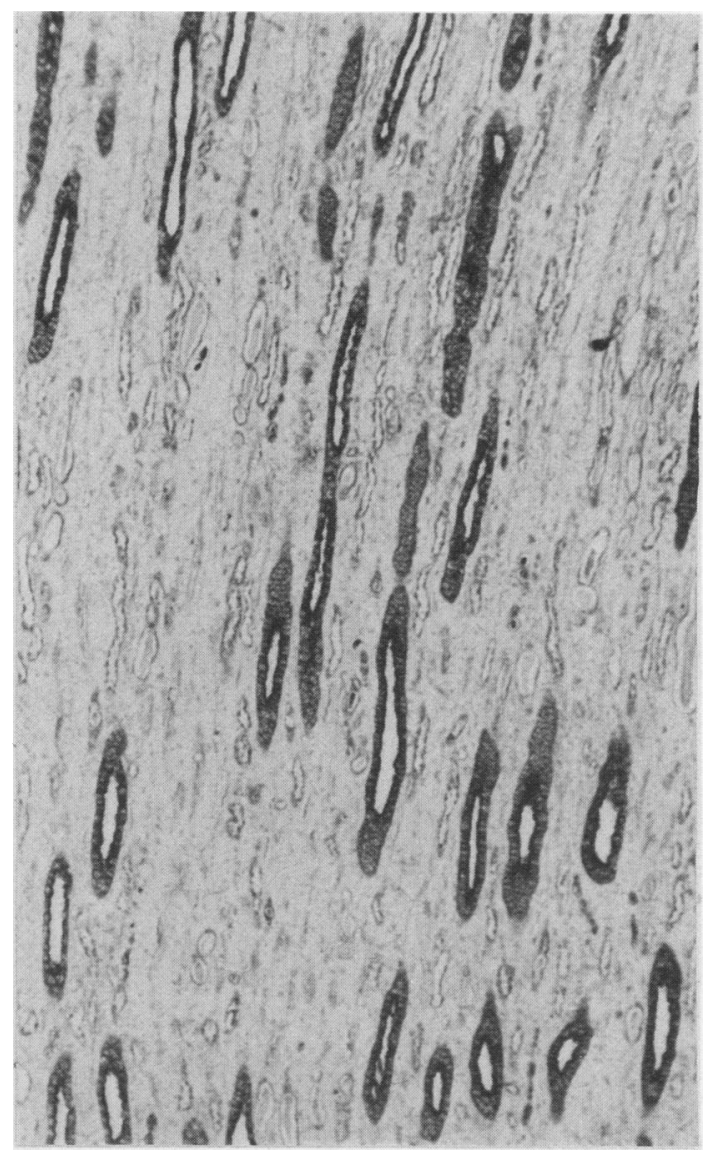

Fig 2 Photomicrograph showing some basic GST positive tubules in medulla.

peroxidase system. ${ }^{16}$ Slides were lightly counterstained with haematoxylin.

\section{Results}

All the kidneys examined were histologically normal. All four GST isoenzymes were identified but their distribution differed (table).

\section{BASIC GST}

This stained both cytoplasm and nuclei in the same cells. There was no staining in glomeruli. Proximal convoluted tubules stained strongly and some medullary tubules, possibly thin loops of Henle also stained (figs 1 and 2). Distal tubules did not stain. Blood vessels did not contain basic GST.

\section{ACIDIC GST}

This showed predominantly cytoplasmic localisation. 


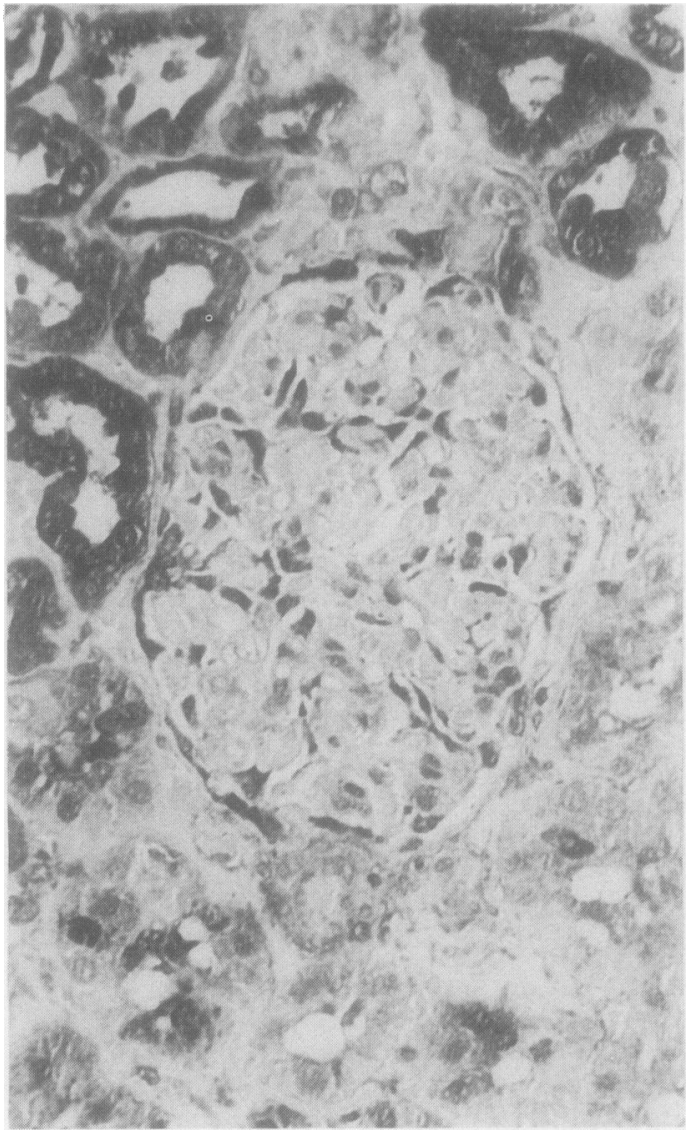

Fig 3 Acidic GST localised predominantly in distal tubules, podocytes, and parietal epithelium of Bowman's capsule.

Most podocytes stained for acidic GST, as well as a variable proportion of the parietal epithelial cells of Bowman's capsule (fig 3). Proximal tubules were only weakly stained, contrasting with the strong staining in distal convoluted tubules and medullary tubules, including collecting ducts (fig 4). Smooth muscle in arterial walls stained moderately.

NEUTRAL GST

The pattern of staining was similar to that seen for acidic GST but was weaker. Distal convoluted tubules were the most strongly stained (fig 5). Reactivity was generally cytoplasmic and the intensity of staining varied between cases. Vasculature did not stain consistently.

MICROSOMAL GST

This stained the cytoplasm. Glomerular and interstitial endothelium were stained but there was only equivocal staining of cortical tubules (fig 6). Collecting ducts deep in the medulla stained (fig 7). There was considerable variation in staining intensity among cases.

\section{Discussion}

The presence of all the major forms of cytosolic GST in human kidney ${ }^{1213}$ was confirmed in this study in addition to the localisation of basic GST to proximal convoluted tubules. ${ }^{7}$ We have also shown the presence of microsomal GST. Its distribution is focal, occurring in endothelium and cellular ducts. Furthermore, we have shown that acidic GST is present in podocyte, parietal epithelium, and distal tubules. The variation in staining intensity for neutral and microsomal GST is of interest as around $40 \%$ of subjects fail to express neutral GST in liver. ${ }^{17}$ In a biochemical study, however, Tateoka et al failed to show the presence of neutral GST in any of five kidneys studied. ${ }^{18}$ It may be that the antibody is cross reacting with another

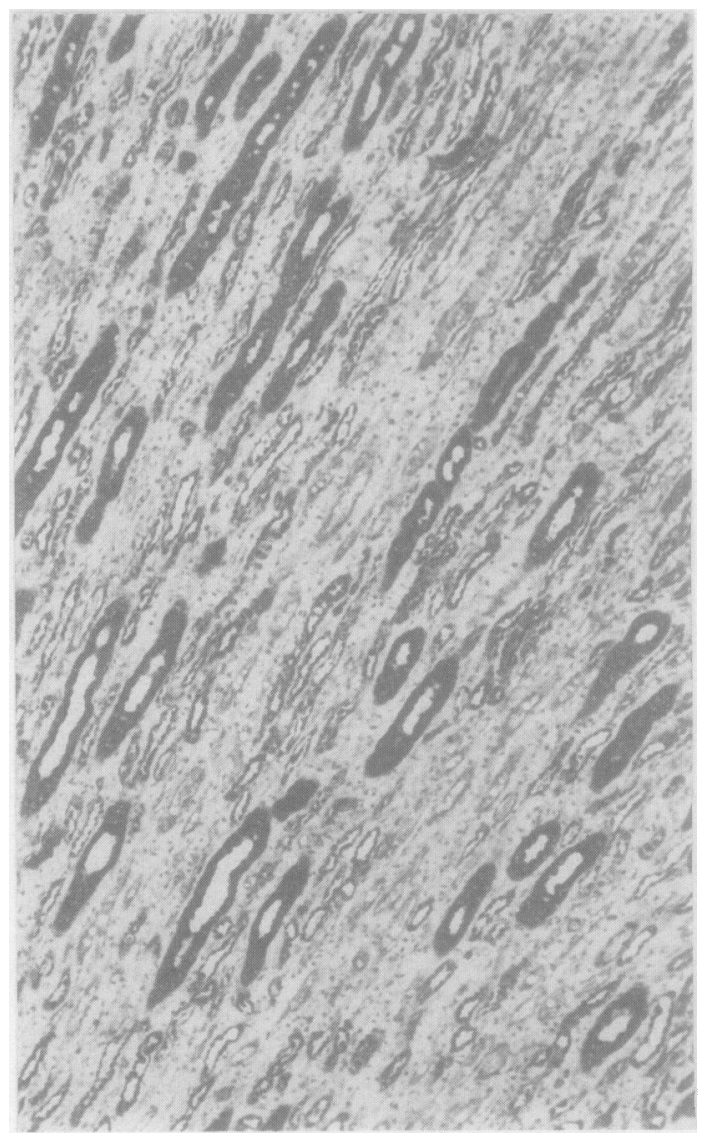

Fig 4 Acidic GST is present in most medullary tubules. 


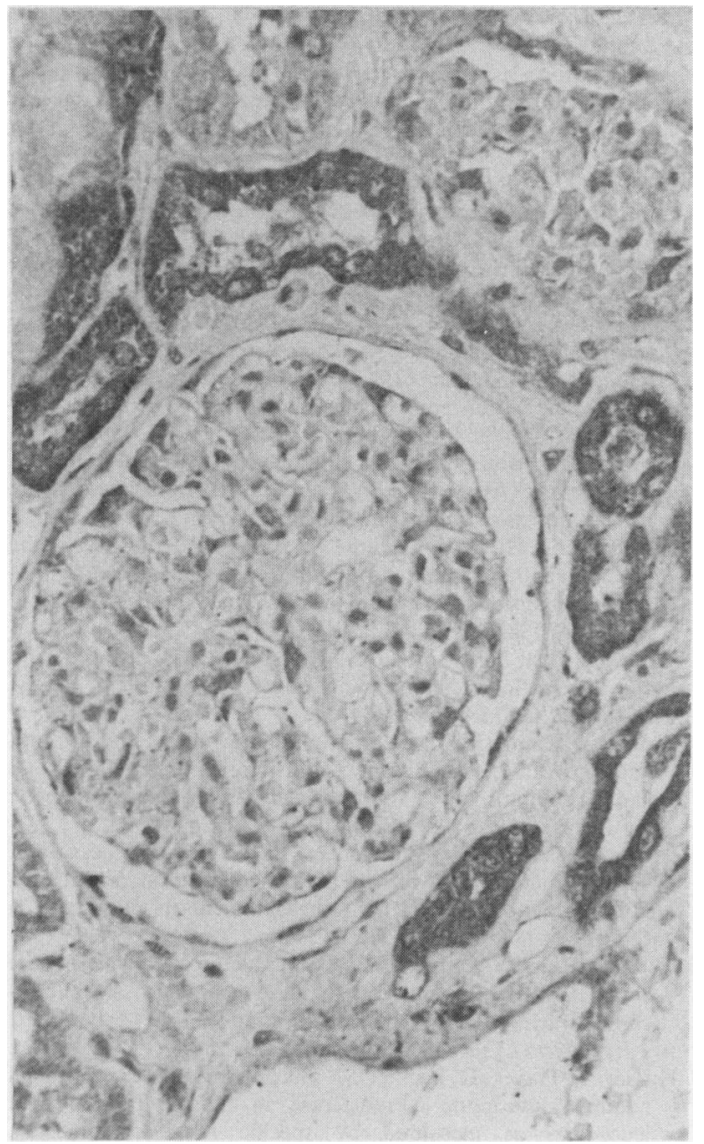

Fig 5 Neutral GST in distal tubules.

molecule, although it gives a single band in Western blots of human liver. These isoenzymes, therefore, are unlikely to be of value as markers of tissue injury.

In many conditions in which tubular damage can occur-for example, septicaemia - there may also be disturbance of hepatic function. Because basic GST is the predominant form of GST shown by immunocytochemistry in the liver, ${ }^{19}$ serum estimations of basic GST alone will not differentiate between liver and kidney injury. If in addition to basic GST, however, the release of acidic GST into serum is high, it may indicate renal tubular injury because acidic GST in the liver is only present in biliary epithelium. ${ }^{19}$ Urine analysis may be more helpful because many forms of tubulotoxin cause basic GST to be released ${ }^{8101}$; glomerular disorders do not result in an increase in urinary GST. ${ }^{20}$

If the urinary excretion of basic GST is high as a result of renal injury, and acidic GST excretion is low, then it is likely that damage is restricted to the proximal convoluted tubule. The presence of both acidic and basic GST in urine may reflect more widespread tubular and even glomerular damage. This may therefore be of diagnostic value in distinguishing between cyclosporine toxicity and transplant rejection, because cyclosporine is though to be primarily a proximal tubule toxin. ${ }^{21}$ Further studies are in progress using radioimmunoassay to measure GST release into serum and urine, to investigate whether GST may be useful in the monitoring of posttransplant kidney allograft rejection.

We are grateful to Dr G Beckett for the gift of antibodies to GST isoenzymes. This work was supported by a grant from the British Medical Association to DJH and Scottish Home and Health Department Grant K/MRS/50/C570 to JDH. RK was supported by an SHHD Student Vacation Grant.

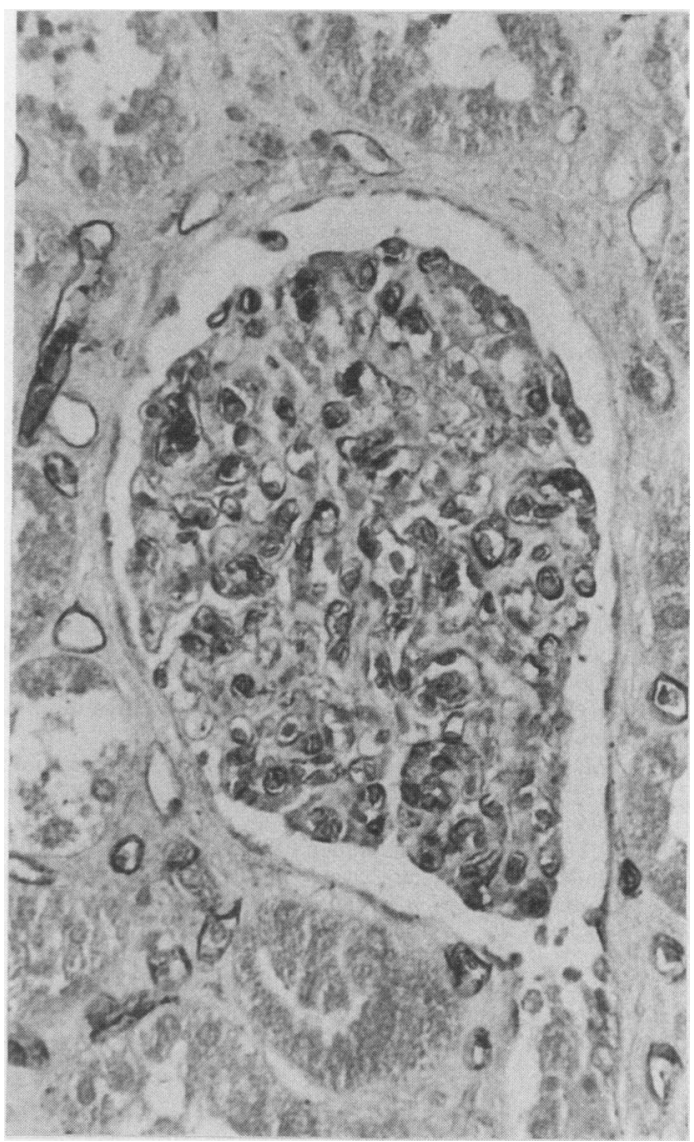

Fig 6 Staining of glomerular and peritubular capillary endothelium for microsomal GST. Red blood cells are also stained. 
628

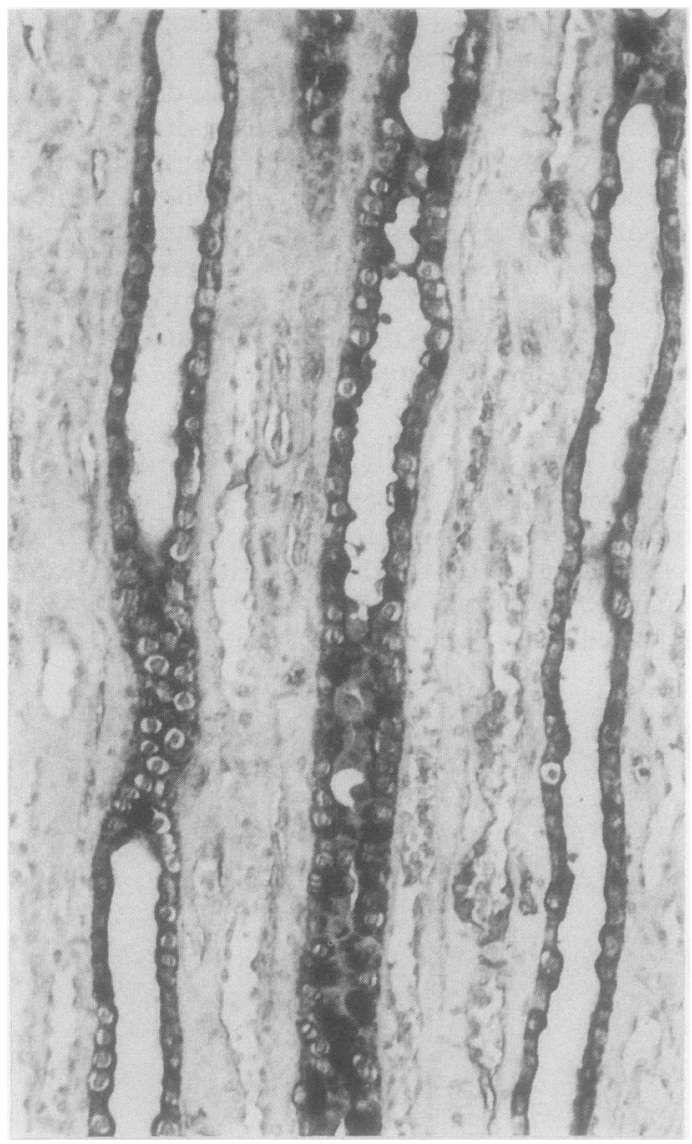

Fig 7 Strong staining of collecting ducts for microsomal GST.

\section{References}

1 Jakoby WB. The glutathione S-transferases: a group of multifunctional detoxification proteins. Adv Enzymol Rel Areas Mol Biol 1977;47:383-414.

2 Mannervik B. The isoenzymes of glutathione S-transferase. $A d v$ Enzymol Rel Areas Mol Biol 1987;57:357-417.

3 Pabst MJ, Habig WH, Jakoby WB. Mercapturic acid formation: The several glutathione transferases of rat liver. Biochem Biophys Res Commun 1973;52:1123-8.

4 Hayes JD; McLellan LI, Stockman PK, Chalmers J, Beckett GJ. Glutathione S-transferases in man: the relationship between rat and human enzymes. Biochem Soc Trans 1987;15:721-5.
Harrison, Kharbanda, Cunningham, McLellan, Hayes

5 McLellan LI, Wolf CR, Hayes JD. Human Microsomal glutathione S-transferase: its involvement in the conjugation of hexacholoro-1, 3-butadiene. Biochem J 1989;258:87-93.

6 Fine LG, Goldstein EJ, Trizna W, Rozmaryn L, Arias IM. Glutathione S-transferase Activity in the Rabbit Nephron. Proc Soc Exp Biol Med 1978;157:189-93.

7 Campbell JAH, Bass NM, Kirch RE. Immunohistological localisation of ligandin in human tissues. Cancer 1980;45:503-10.

8 Feinfeld DA, Fuh UL. Urinary glutathione-S-transferase in cisplatin nephrotoxicity in the rat. Clin Chem Clin Biochem 1986;24:529-32.

9 Zalneraitis B, Ariao IM, Cho SI. Prediction of cadaver kidney function by ligandin analysis. Transplant Proc 1981;13:697-8.

10 Feinfeld DA, Fleischner GM, Arias IM. Urinary ligandin and glutathione S-transferase in gentamicin-induced nephrotoxicity in the rat. Clin Sci 1981:123-5.

11 Feinfeld DA, Bourgoignie JJ, Fleischner GM, Goldstein EJ, Biempica L, Arias IM. Ligandinuria in nephrotoxic acute tubular necrosis. Kidney Int 1977;12:387-92.

12 Fauldner CG, Hirrell PA, Hume R, Strange RC. Studies of the development of basic, neutral and acidic isoenzymes of glutathione S-transferase in human liver, adrenal, kidney and spleen. Biochem J 1987;241:221-8.

13 Singh SV, Leal T, Ansari GAS, Awasthi YG. Purification and characterisation of glutathione S-transferases of human kidney. Biochem J 1987;246:179-86.

14 Hayes JD, Mantle TJ. Use of immunoblot techniques to discriminate between the glutathione S-transferase Yf, Yk, Ya, Yn/Yb and Yc subunits and to study their distribution in extrahepatic tissues. Biochem J 1986;233:779-88.

15 Hayes JD, Gilligan D, Chapman BJ, Beckett GJ. Purification of hepatic glutathione S-transferases and the development of a radio-immunoassay for their measurement in plasma. Clin Chim Acta 1938;134:107-21.

16 Hsu SM, Rame L, Fanger H. Use of an avidin-biotin-peroxidase complex $(\mathrm{ABC})$ in immunoperoxidase techniques. A comparison between $\mathrm{ABC}$ and unlabelled antibody (PAP) procedures. J Histochem Cytochem 1981;29:577-83.

17 Hussey AJ, Hayes JD, Beckett GJ. The polymorphic expression of neutral glutathione S-transferase in human mononuclear leucocytes as measured by specific radio-immunoassay. Biochemical Pharmacology 1987;36:4013-15.

18 Tateoka N, Tsuchida S, Soma Y, Sato K. Purification and characterisation of glutathione S-transferases in human kidney. Clin Chim Acta 1987;166:207-18.

19 Hayes PC, Harrison DJ, Bouchier IAD, McLellan LI, Hayes JD. Cytosolic and microsomal glutathione S-transferase isoenzymes in normal human liver and intestinal epithelium. Gut (in press).

20 Feinfeld DA, Fleischner GM, Goldstein EJ, et al. Current Problems in Clinical Biochemistry 1979;9:273-80.

21 Whiting PH, Thomson AW, Blair JT, Simpson JG. Experimental cyclosporine A reprotoxicity. Br J Exp Pathol 1982;63:88-94.

Requests for reprints to: Dr D J Harrison, Department of Pathology, University of Edinburgh Medical School, Teviot Place, Edinburgh EH8 9AG, Scotland. 